\title{
Functional characterization of Fas ligand on tumor cells escaping active specific immunotherapy
}

\author{
D Céfai ${ }^{1,3}, R$ Schwaninger ${ }^{1,3}, M$ Balli ${ }^{2}, T$ Brunner $^{2}$ and \\ CD Gimmi ${ }^{*, 1}$ \\ 1 Department of Clinical Research, University of Bern, 3010 Bern, Switzerland \\ 2 Institute of Pathology, University of Bern, 3010 Bern, Switzerland \\ ${ }^{3}$ Both authors contributed equally to this work \\ * Corresponding author: CD Gimmi, Department of Clinical Research, University \\ of Bern, 35 Murtenstrasse, 3010 Bern, Switzerland. Tel: 413163287 77; \\ Fax: 413163232 97; E-mail: gimmi@dkf5.unibe.ch
}

Received 29.9.00; revised 5.1.01; accepted 13.2.01

Edited by KM Debatin

\begin{abstract}
Mice transgenic for the rat HER-2/neu oncogene (rNeu-TG) developed spontaneous breast tumors that can escape a rNeu-specific immune response induced by active specific immunotherapy (ASI). The ability of these escape tumors to grow appeared to be due to upregulation of the Fas ligand (FasL) molecule. In an effort to develop tools for the better elucidation of the role of Fas-L and other regulatory mechanisms in tumor escape, we established cell lines derived from escape tumors. These tumor cell lines retained MHC class I, rNeu and Fas-L expression in vitro and formed tumors in vaccinated mice. Tumor growth was accompanied by permanent Fas-Lexpression in vivo, both in vaccinated and control vaccinated mice, indicating that these cells have acquired constitutive Fas-L expression. Moreover, these cells induced target cell apoptosis in vitro. Thus, these cells represent a unique tool to elucidate the importance of Fas-L expressed by tumors that escaped efficient systemic immune responses. Cell Death and Differentiation (2001) 8, 687-695.
\end{abstract}

Keywords: HER-2/neu; tumor cell lines; immune escape; Fas ligand

Abbreviations: ASI, active specific immunotherapy; TAAG, tumorassociated antigens

\section{Introduction}

The identification of tumor-associated antigens (TAAg) expressed on tumor cells has allowed the development of active specific immunotherapy (ASI) protocols. TAAg-specific immune responses have demonstrated rejection and/or regression of various established tumors in animal models. However, numerous studies have indicated that tumors can develop passive and/or active escape mechanisms to evade the immune system in immunized animals. ${ }^{1}$ Loss of TAAg, downregulation of MHC molecules, ${ }^{2-4}$ lack of costimulatory molecules ${ }^{5-7}$ and upregulation of immune-suppressive factors have been reported. ${ }^{8-10}$

Recent reports have identified Fas (CD95, APO-1) ligand (Fas-L) as a molecule participating in tumor resistance to the immune system. Human tumors such as melanoma ${ }^{9}$ hepatoma,${ }^{11}$ gastro-intestinal cancer, ${ }^{10,12,13}$ lung cancer ${ }^{14}$ and breast carcinoma ${ }^{15-18}$ have been shown to express Fas- $L$ and to induce apoptosis of $T$ cells in vitro. Apoptosis of tumor infiltrating lymphocytes was found in areas of Fas$L$ expressing tumor cells. ${ }^{12}$ These observations led to the hypothesis of tumor counterattack by which Fas-L expressing cancers might delete tumor-specific lymphocytes and promote evasion from an anti-tumor immune response. ${ }^{19,20}$ In contrast, other reports demonstrated that cells engineered to express high levels of Fas- $L$ did not confer immune privilege but rather induced a proinflammatory granulocytic infiltration that resulted in rapid elimination of the transplanted cells. ${ }^{21-24}$ These contradictory results indicated that the role of Fas-L to promote tumor escape in vivo needed to be further validated. In particular, no study so far has conclusively demonstrated that a tumor constitutively expressing Fas-L could delete specific anti-tumor lymphocytes and escape vaccinationinduced immune rejection.

Transgenic mice (rNeu-TG) in which the rat oncogene HER-2/neu (rNeu) is overexpressed in the mammary gland epithelium develop spontaneous breast tumors after a first pregnancy. ${ }^{25}$ We have previously shown that a vaccine consisting of allogeneic fibroblasts overexpressing $\mathrm{rNeu}$ (Balbc/neu) prevented spontaneous tumor formation while late escape tumors developed in some vaccinated mice. ${ }^{26}$ Our data further showed that escape tumors upregulated Fas-L in mice with a persistent rNeu-specific systemic immune response and indicated that Fas- $L$ expression promoted $\mathrm{T}$ cell apoptosis in situ and enabled tumor escape. $^{27}$

Tumor cell lines represent a widely used tool to investigate the role of Fas- $L$ in tumor escape. Tumor rejection was mainly observed when Fas-L-transfected cells were used and suggested Fas-L (over) expressing cells to be responsible for non-specific immunologic reactions such as the documented granulocytic infiltrations. ${ }^{21-24}$ However, cell lines derived from Fas-L-expressing escape tumors may offer more reliable models to assess Fas-L involvement in tumor escape from ASI. We established and characterized here tumor cell lines derived from Fas $-\mathrm{L}^{+}$ escape tumors that developed in vaccinated rNeu-TG mice. We showed that these cell lines maintained rNeu, MHC class I and Fas- $L$ expression in vitro, formed tumors in Balbc/neu vaccinated mice and induced Fas-L-dependent target cell apoptosis in vitro. Thus, these cell lines represent a unique tool to investigate the mechanism of Fas-L-dependent tumor escape after ASI in vivo. 


\section{Results}

Tumor cells derived from escape tumors maintain their ability to grow in vaccinated mice

We investigated whether tumor cells that escaped from an efficient immune response after ASI would maintain their tumor formation capacity after transplantation into vaccinated mice. Cell suspension from one representative escape tumor (mouse \#241) was reinjected in vaccinated mice as shown in Figure 1. Escape tumor cells formed tumors at the injection sites irrespective of whether transplanted in mice vaccinated with control allogeneic fibroblasts (Balbc/mock) or with fibroblasts transfected with $\mathrm{rNeu}$ (Balbc/neu). ${ }^{26}$ These escape properties were maintained when newly formed tumors (from mouse \#490) were passaged into a Balbc/neu vaccinated mouse (mouse \#557). In contrast, Balbc/neu vaccinated mice repetitively rejected a challenge with a cell suspension of spontaneous tumor cells derived from an unvaccinated mouse (e.g. mouse \#345), although these cells remained tumorigenic in Balbc/mock vaccinated mice. We have previously shown that most escape tumors expressed Fas-L on their cell surface. ${ }^{27}$ To investigate whether Fas-L expression was maintained in passaged escape tumors we assessed Fas-L expression by immunohistochemical staining. All but one (\#491) tumors that developed from escape tumor \#241 expressed significant levels of Fas-L. Identical results were obtained with different transplanted escape tumors (not shown). In contrast, spontaneous tumors transplanted in Balbc/mock vaccinated mice did not upregulate Fas- $L$ expression. These results indicated that escape properties and Fas-L expression were maintained after transplantation of escape tumors in vaccinated mice.

\section{Phenotypic characterization of an escape tumor cell line}

To further assess the function of naturally occurring Fas- $L$ on tumor cells that escaped ASI, we generated stable cell lines from spontaneous and escape tumors. Two representative cell lines derived from a spontaneous (NF9006) and as escape tumor (\#493) were further analyzed. Both tumor cell lines grew in vitro as a monolayer with an epithelial-like morphology and stained positive for pan-cytokeratin (not shown). As depicted in the FACS histograms in Figure 2A, NF9006 and \#493 cells expressed similar levels of rNeu. In contrast, the \#493 escape tumor cell line expressed higher levels of $M H C$ class I (H-2 $D^{q} L^{q}$, IgG2a) molecules. Both NF9006 and \#493 cell lines were negative for MHC class II (I$A^{\mathrm{q}}$, IgG2a isotype-matched control) and for CD80 and CD86 (not shown). Fas-L surface expression was assessed using a $\mathrm{mAb}$ recognizing the extracellular part of murine Fas-L (IgG2a). The escape tumor cell line \#493 expressed elevated levels of Fas-L on the cell surface in comparison to the weak Fas-L levels on NF9006 cells. Previous studies have reported inconsistency in Fas-L detection by flow cytometric analysis depending on the mAb used. ${ }^{28}$ To further confirm increased Fas-L protein production in \#493 cells, immunohistochemical staining was performed on cytospins using a rabbit polyclonal antibody against the intracellular part of Fas-L (Figure 2B). The spontaneous tumor cell line NF9006 showed only a faint staining for Fas-L while significant Fas-L protein level was detected in the cytoplasm and membrane of \#493 cells. We also performed RT-PCR for Fas-L on total RNA extracted from NF9006 and \#493 tumor cells. No fragment was amplified from RNA extracts without reverse transcriptase ruling out DNA contamination. The resulting PCR product was the expected size of 547 bp (data not shown) and showed an identical sequence as the reported murine Fas-L. ${ }^{29}$

\section{The escape cell line \#493 maintains the capacity of tumor formation in Balbc/neu vaccinated mice}

We then investigated whether the \#493 tumor cell line expressing Fas-L and cultured in vitro maintained its escape capacity in vivo. NF9006 and \#493 cells $\left(0,5-2 \times 10^{6}\right)$ were injected into Balbc/mock or Balbc/neu-vaccinated mice and
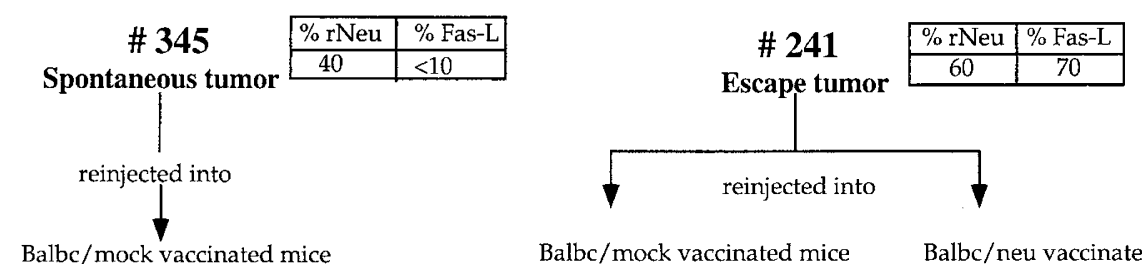

Balbc/mock vaccinated mice

Balbc/mock vaccinated mice Balbc/neu vaccinated mice

\begin{tabular}{|c|c|c|}
\hline mouse & $\%$ rNeu & $\%$ Fas-L \\
\hline 511 & 40 & 10 \\
\hline 572 & 70 & $<10$ \\
\hline 569 & $\mathrm{na}$ & n a \\
\hline 570 & 40 & negative \\
\hline
\end{tabular}
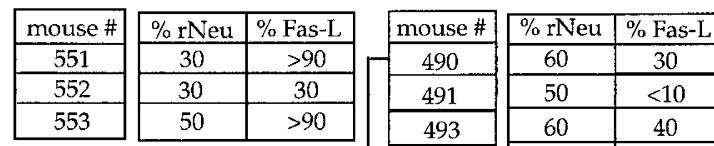

\begin{tabular}{|c|c|c|}
\hline mouse \# & $\%$ rNeu & $\%$ Fas-L \\
\hline 490 & 60 & 30 \\
\hline 491 & 50 & $<10$ \\
\hline 493 & 60 & 40 \\
\hline 494 & 40 & 70 \\
\hline mouse \# & $\%$ rNeu & $\%$ Fas-L \\
\hline$>557$ & 80 & 80 \\
\hline
\end{tabular}

Figure 1 Cell lines derived from escape tumors maintain the escape properties of their originating tumor. Cell suspensions $\left(2 \times 10^{6}\right)$ derived from spontaneous breast tumor \#345 or from escape breast tumor \#241 were injected s.c. into Balbc/mock or Balbc/neu vaccinated mice, as indicated. Tumor formation was monitored at the injection site. Where indicated, $2 \times 10^{6}$ tumor cells derived from mouse \#490 was reinjected into a Balbc/neu vaccinated mouse. The results of $r$ $\mathrm{Neu}$ and Fas-L expression (per cent of positive cells), as assessed by three independent immunohistochemical stainings with specific Abs are shown for each tumor. (na, not assessed) 
time to tumor formation and kinetics of tumor growth were assessed. As shown in Table 1, tumors formed in 12 out of 15 Balbc/neu vaccinated mice challenged with \#493 escape tumor cells. There was no significant difference in the time to tumor formation between Balbc/mock (mean 23.8 days, S.E.M. 3.6) and Balbc/neu (mean 31.2 days, S.E.M. 4.2, $P<0.28)$ vaccinated mice. The growth kinetic was similar in Balbc/neu and Balbc/mock vaccinated mice showing in each

A
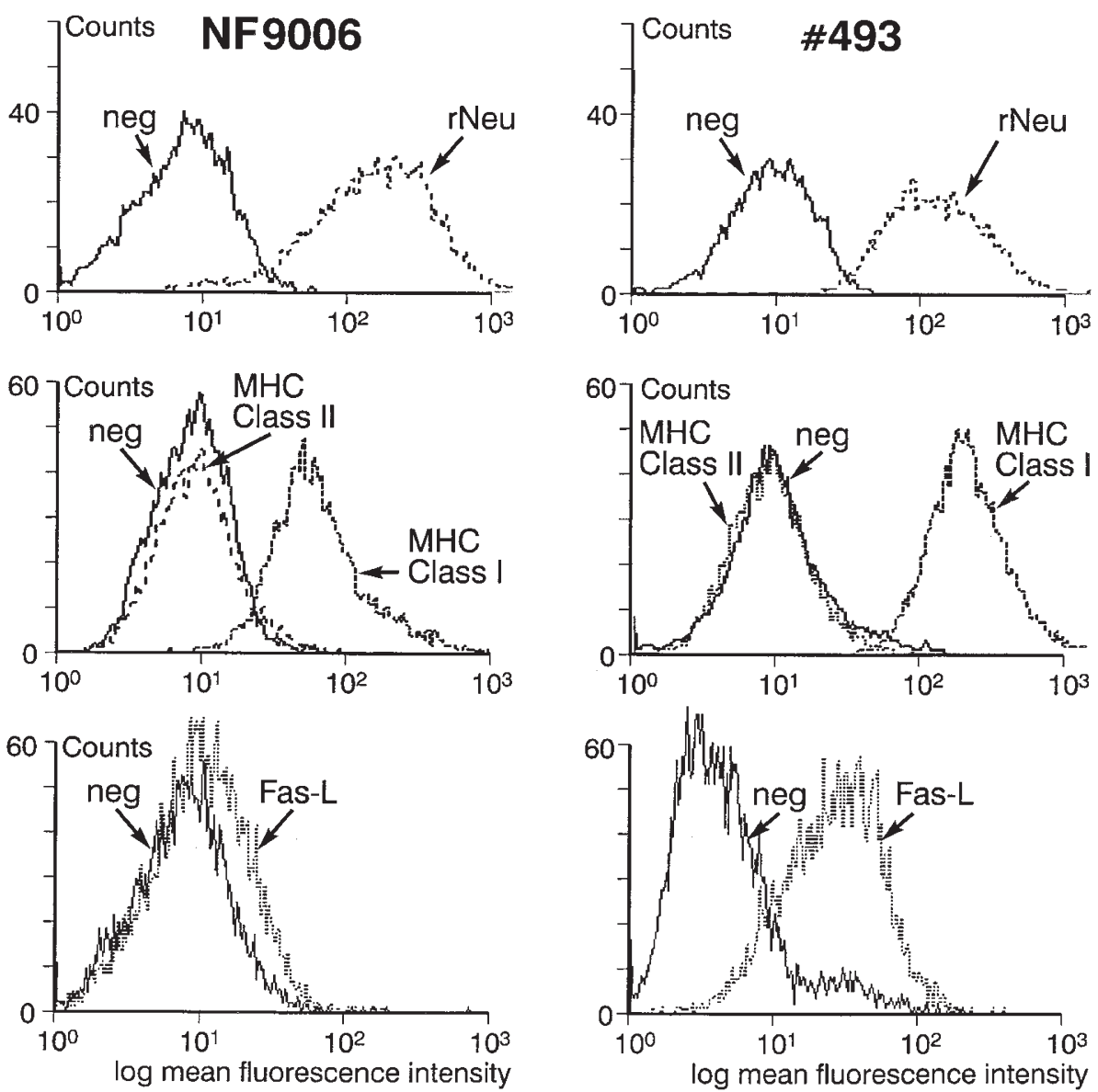

B
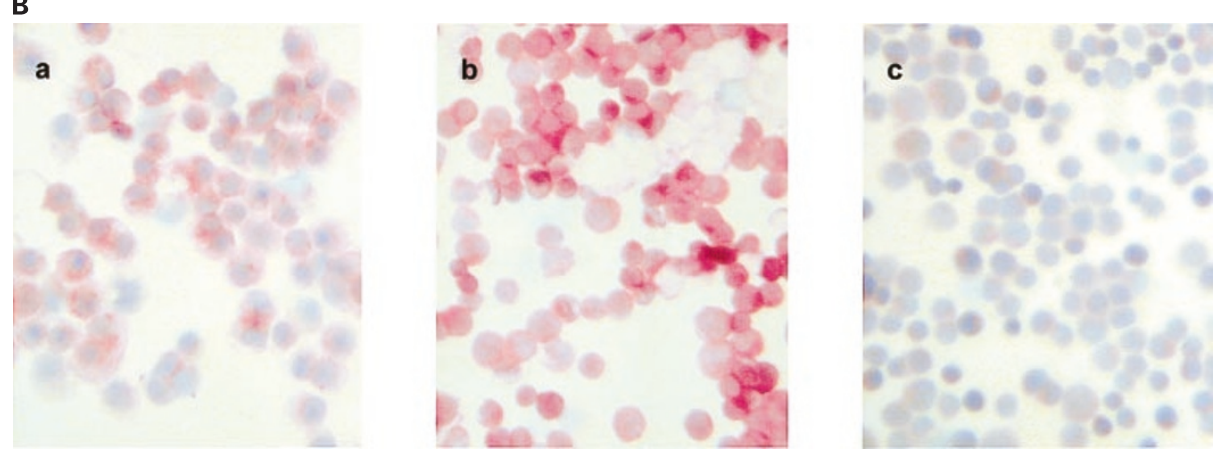

Figure 2 Analysis of Fas-L expression in NF9006 and \#493 tumor cell lines. (A) rNeu ${ }^{+}$spontaneous tumor cell line NF9006 and escape tumor cell line \#493 were analyzed for $\mathrm{rNeu}, \mathrm{MHC}$ class I $\left(\mathrm{H}-2 \mathrm{D}^{\mathrm{q}} / \mathrm{L}^{\mathrm{q}}\right)$, MHC class II $\left(\mathrm{I}-\mathrm{A}^{\mathrm{q}}\right)$ and Fas-L cell surface expression by indirect immunostaining using specific Abs followed by Streptavidin-FITC or FITC-conjugated goat anti-mouse IgG. Control staining (neg) was performed in the absence of corresponding primary Ab. Isotype-matched (IgG2a) control antibody is anti-I-A (MHC class II). Fluorescence was analyzed on a FACScan. (B) Cytospins of NF9006 (a) and \#493 (b) cell lines were analyzed for Fas-L expression by immunohistochemical staining using a Fas-L specific Ab, as described in Materials and Methods. Also shown is a control staining of \#493 cell line (c) using a rabbit anti-Fas primary Ab 
group nine mice with tumors below $1.5 \mathrm{~cm}^{2}$ at day 25 (Figure $3)$. In contrast, nine of 10 mice vaccinated with Balbc/mock exhibited a tumor size of $>1.5 \mathrm{~cm}^{2} 50$ days after challenge, whereas at this time point four Balbc/neu vaccinated mice had tumors $<1.5 \mathrm{~cm}^{2}$ and three mice showed still no tumor at the injection site. At day 50 , mice vaccinated with Balbc/mock

Table 1 Time to tumor formation of injected escape tumor cell lines in vaccinated mice

\begin{tabular}{lcc}
\hline $\begin{array}{l}\text { Injected escape } \\
\text { tumor cells \#493 }\end{array}$ & $\begin{array}{c}\text { Time to tumor formation (days) } \\
\text { Balbc/mock } \\
\text { vaccinated mice }\end{array}$ & $\begin{array}{c}\text { Balbc/neu } \\
\text { vaccinated mice }\end{array}$ \\
\hline $0.5 \times 10^{6}$ cells & 12 & 24 \\
& 30 & 30 \\
& 35 & 26 \\
$1 \times 10^{6}$ cells & 12 & No tumor formation \\
& 38 & 17 \\
& 42 & 16 \\
& & 16 \\
& 12 & 18 \\
& 19 & 43 \\
& 19 & 20 \\
& 19 & 30 \\
& & 24 \\
& & 24 \\
& & No tumor formation \\
& & No tumor formation \\
\hline
\end{tabular}

0.5-2 $\times 10^{6} \# 493$ tumor cells were injected s.c. into Balbc/mock or Balbc/ neu vaccinated mice, as indicated, and tumor growth was monitored at the injection site. Results are expressed as time to first detection of palpable/visible tumor mass fibroblasts showed a mean tumor size of $3.12 \mathrm{~cm}^{2}$ (S.E.M. 0.71 ), whereas mice vaccinated with Balbc/neu cells had a mean tumor size of $1.53 \mathrm{~cm}^{2}$ (S.E.M. 0.3). Tumors formed in mice irrespective of whether they were injected with $0.5,1$ or $2 \times 10^{6}$ tumor cells. However there was a significant delay of tumor growth/size in Balbc/neu vaccinated mice (MannWhitney rank test $P<0.024)$. Moreover, NF9006 formed tumors in all Balbc/mock vaccinated mice and no tumor was observed in Balbc/neu vaccinated mice as described previously. ${ }^{26}$ These experiments showed that the \#493 cells derived from an escape tumor maintained the tumorigenic and escape capacity of the originating tumor.

\section{Injected \#493 cells maintain Fas-L expression in vivo}

To test whether Fas- $L$ was still expressed in vivo in the longterm follow up, \#493 and NF9006 cells were injected into Balbc/neu and Balbc/mock vaccinated mice and expression of Fas- $L$ in situ was assessed by immunohistochemistry at the injection site. As shown in Figure 4A, \#493 cells injected into Balbc/neu vaccinated mice formed disrupted tumor islets by day 5 and most tumor cells stained positive for Fas-L. We have previously shown that escape tumor cells injected in Balbc/neu vaccinated mice were infiltrated by a large number of $\mathrm{CD}^{+}$lymphoid cells in the tumor implant as well as the surrounding tissue. ${ }^{27}$ We further showed that expression of Fas- $L$ on tumor cells correlated with local induction of $T$ cell apoptosis as predominantly, cells in the close periphery of injected escape tumor cells, stained positive for active caspase-3. The regions of caspase-3 activity overlapped

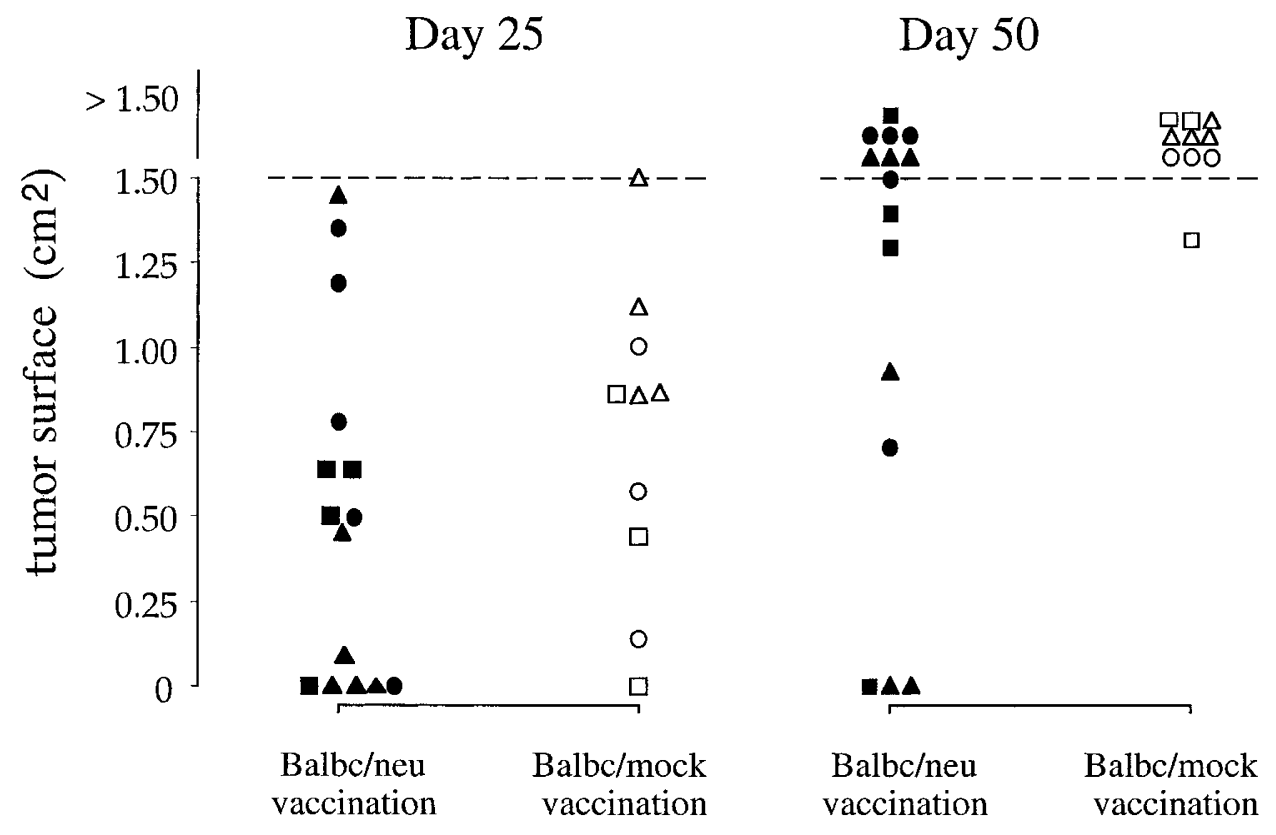

Figure 3 Tumor kinetics of injected escape tumor cell lines in vaccinated mice. Results as in Table 1 are expressed as tumor surface (cm ${ }^{2}$ ) at the injection site at days 25 and 50 after injection. Open symbols are mice vaccinated with Balbc/mock fibroblasts and closed symbols are mice vaccinated with Balbc/neu fibroblasts. Challenge with $0.5 \times 10^{6}$ cells (squares), $1 \times 10^{6}$ cells (circles) and $2 \times 10^{6}$ cells (triangles) 

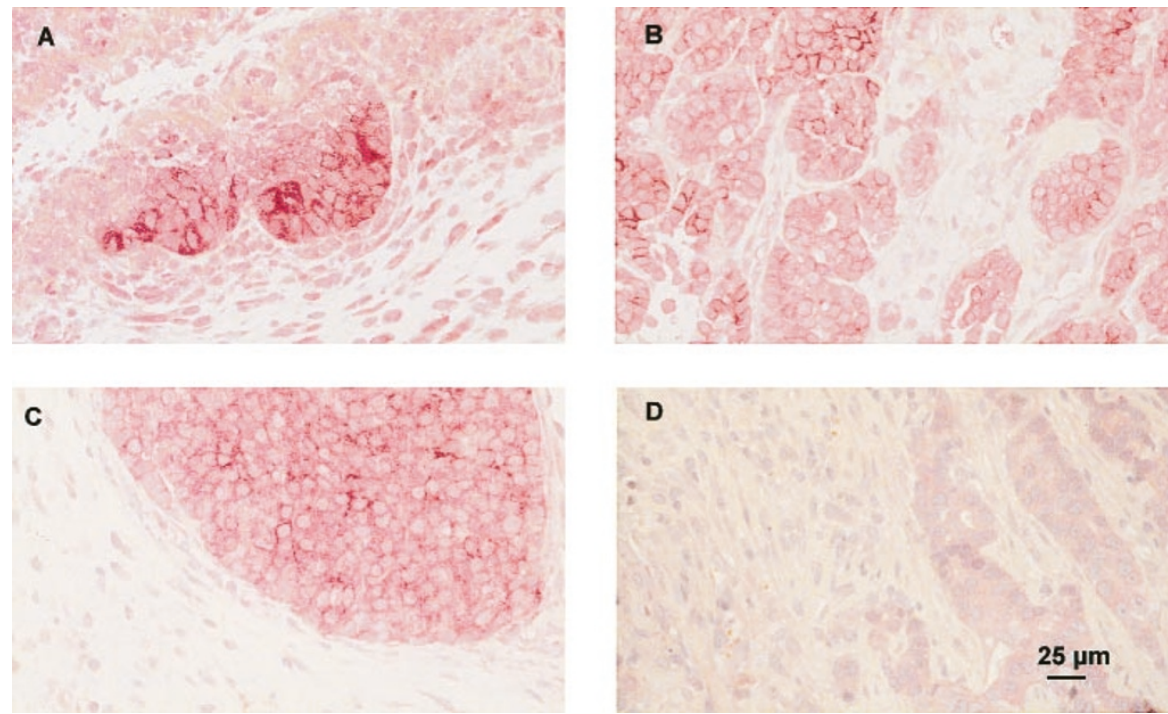

Figure 4 Expression and distribution of Fas-L in injected escape and spontaneous tumor cells. Tumors at days 5 (A) and 30 (B) after injection of \#493 escape tumor cells into Balbc/neu-vaccinated mice were analyzed for Fas-L expression by immunohistochemical staining with specific Ab. Also shown are Fas- $L$ stainings of \#493 tumor cells (C) and NF9006 tumor cells (D) 30 days after injection into Balbc/mock vaccinated mice. Magnification $\times 40$ (Bar represents $25 \mu \mathrm{m}$ )

with the localization of $\mathrm{CD}^{+}$lymphocytes. At day 30 after challenge, tumors maintained a marked Fas- $L$ staining (Figure 4B). No $T$ cell infiltration was seen in close proximity of escape tumors at this time point. The same intense Fas- $L$ expression was also detected in tumors of Balbc/mock vaccinated mice after challenge with \#493 (Figure 4C). NF9006 cells that formed tumors in Balbc/mock vaccinated mice stained negative for Fas- $L$ throughout the observation time and no tumor developed in Balbc/neu vaccinated mice challenged with NF9006 cells (not shown). These results indicated that \#493 cells maintained Fas-L expression in vivo in the presence of a TAAg-specific immune response and that the allogeneic immune response induced by Balbc/mock cells did not cause Fas-L expression on spontaneous tumor cells.

\section{Escape tumor cell lines induce apoptosis of Fas-L-sensitive target cells}

We next investigated whether Fas-L expressed on tumor cells might be functionally responsible for the induction of apoptosis of infiltrating $\mathrm{T}$ cells. ${ }^{27}$ We used the P815 mastocytoma cell line transfected with murine Fas cDNA as Fas-L-sensitive target cells in a modified cytotoxicity test. ${ }^{30,31}$ P815-Fas cells have been used as appropriate target cells to investigate putative Fas- $L$ activity on tumor cells, as no Fas- $L$ is expressed on P815 wild type cells (P815-WT, Calzascia personal communication). P815-WT and P815-Fas were cultured either alone or together with NF9006 or \#493 cells. The data depicted in Figure 5A demonstrated that \#493 tumor cells mediated a cell density dependent killing of P815-Fas cells reaching approximately $40 \%$ lysis at $E: T$ ratio of 16 while no DNA fragmentation was observed in P815-WT. In contrast, the spontaneous tumor cell line NF9006 did not induce significant DNA fragmentation neither of P815-Fas nor of P815-WT cells. In addition, we observed the same dosedependent cytotoxicity with a different escape tumor cell line (\#242, data not shown). To further support the finding that Fas-L on \#493 cells was responsible for DNA fragmentation of P815-Fas cells, cocultures were performed in the presence of Fas-Fc fusion protein $(20 \mu \mathrm{g} / \mathrm{ml})$ to block the Fas/Fas-L interaction. The data shown in Figure 5B indicated that cytotoxicity induced by \#493 was mainly mediated by Fas-L as the addition of Fas-Fc fusion protein inhibited DNA fragmentation. The same inhibition of DNA fragmentation was observed when \#242 escape tumor cells were used as effector cells and Fas-Fc protein was added to the culture system (data not shown). In contrast, Fas-Fc fusion protein had no effect on cell cultures with P815-WT or in coculture systems with NF9006 as effector cells. Thus, the capacity of Fas-L expressing \#493 cells to kill Fas-sensitive target cells and its inhibition by Fas-Fc fusion protein strongly suggests a role of constitutively expressed Fas- $L$ on tumor cells as an active tumor escape mechanism.

\section{Discussion}

We have previously shown that some rNeu-TG mice developed late escape mammary tumors after a rNeuspecific vaccination with Balbc/neu fibroblasts. ${ }^{27}$ These escape tumors expressed elevated levels of surface Fas-L that appeared to promote tumor escape from rNeu specific immune response after vaccination. This created a unique opportunity for the isolation of cell lines that constitutively expressed Fas-L. We showed here that cell lines derived from $\mathrm{rNeu}^{+}$escape tumors maintained the Fas-L expression of the original tumor and were tumorigenic in Balbc/neu vaccinated mice, in contrast to a tumor cell line derived from a spontaneous tumor that developed in unvaccinated mice. 

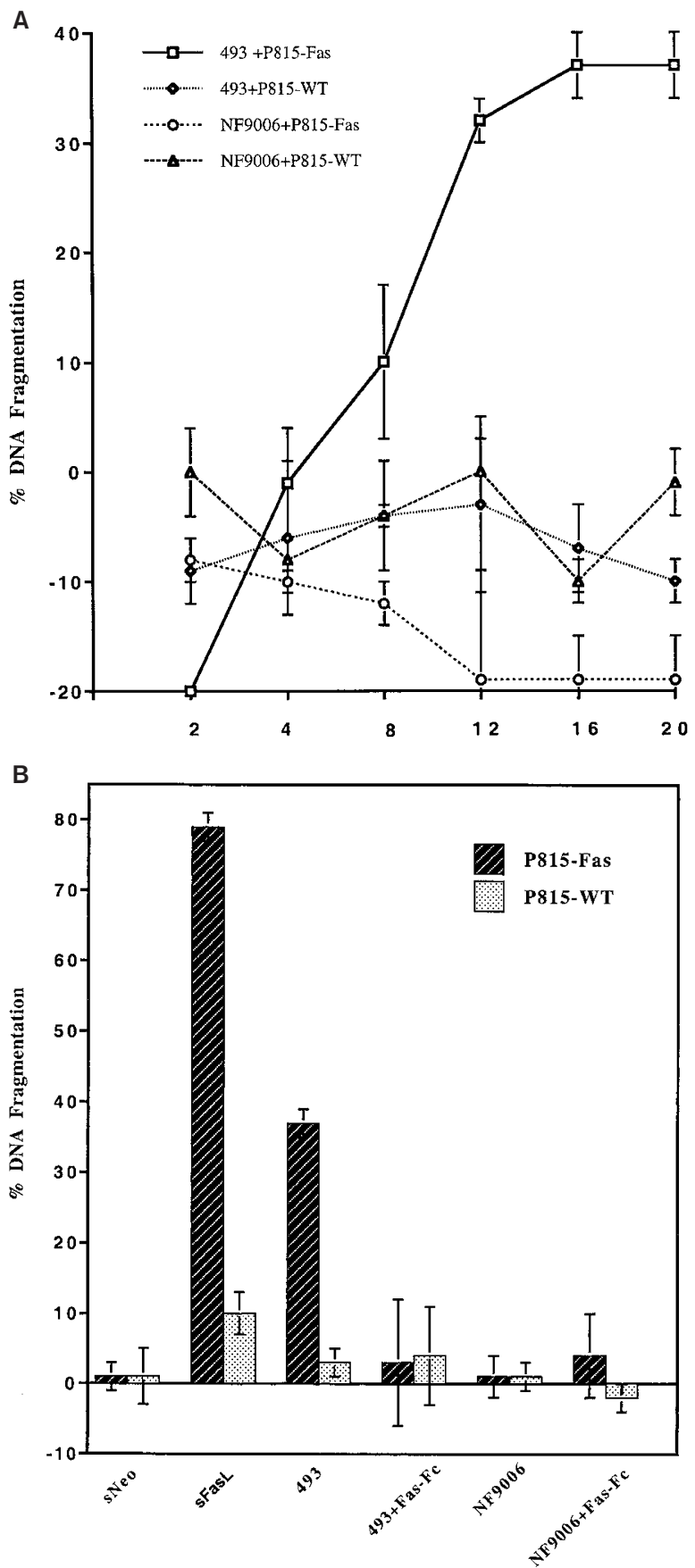

Figure 5 Fas-L expressed on \#493 escape cells mediates DNA fragmentation in Fas ${ }^{+}$P815 mastocytoma cells. (A) P815-Fas and P815-WT cells were labeled with ${ }^{3} \mathrm{H}$-thymidine and used as targets to study the ability of \#493 escape tumor cells to mediate cell death in comparison to the spontaneous tumor cell line NF9006. Target cells were used at a concentration of 5000 cells/well. Different effector: target ratios $(E: T)$ were investigated as indicated. Results are expressed as \% DNA fragmentation in relation to target cells in the absence of effector cells. Vertical bars represent mean \pm s.d. in triplicate cultures. (B) ${ }^{3} \mathrm{H}$-thymidine labeled P815-Fas and P815-WT cells (5000 cells/ well) were cultured in vitro for $8 \mathrm{~h}$ in the presence of Neo supernatant (sNeo, $1: 4=v: v)$, supernatant from Neuro-2a cells stably transfected with Fas-L (sFas-L, 1:4=v:v), or cocultured with 60000 adherent \#493 escape or NF9006 spontaneous tumor cells. Fas-Fc fusion protein was added at a concentration of $20 \mu \mathrm{g} / \mathrm{ml}$ as indicated. Results are expressed as in A. This is one representative experiment of three performed with similar results
Moreover, we observed Fas- $L$ expression throughout the in vivo tumor growth, both in Balbc/mock and Balbc/neu vaccinated mice. Escape tumor cell lines induced a Fasdependent target cell apoptosis in vitro, thus demonstrating the functional activity of cell surface Fas-L.

Fas- $L$ plays a pivotal role in lymphocyte cytotoxicity and in the maintenance of immunologic homeostasis ${ }^{32}$ and has been implicated in immune privilege of various organs, such as the eye and testis by inducing apoptosis of activated $\mathrm{T}$ lymphocytes. ${ }^{33,34}$ Immune privilege has also become a new focus in tumor immune evasion, as various types of cancer cells including breast cancer, ${ }^{15-18}$ hepatocellular carcinomas, ${ }^{11}$ colon cancer $^{10}$ and melano$\mathrm{ma}^{9}$ have been described to express Fas-L. The functional activity of tumor-derived Fas- $L$ was demonstrated in vitro by the induction of apoptosis of activated T cells and in vivo by the detection of apoptotic $T$ cells in the vicinity of Fas- $L$ expressing tumors. ${ }^{9-14}$ We have shown in our rNeu-TG mouse model a predominant expression of Fas- $L$ on escape tumor cells. We further demonstrated here that Fas- $L$ also expressed on a representative escape tumor cell line that formed tumors in vaccinated mice. Moreover, we previously described apoptotic $\mathrm{T}$ cells in close proximity of Fas- $L$ expressing escape tumors, in contrast to Fas- $L$ negative spontaneous tumors. ${ }^{27}$

However, the initial observations of Fas-L expression on melanoma cells and the induction of apoptosis of activated T cells ${ }^{9}$ were not confirmed by subsequent studies, ${ }^{21,35}$ putting Fas- $L$ in question as a tumor counterattack mechanism. $^{20,36}$ Fas- $L$ detection on some tumor samples has suffered from technical inconsistencies based on unspecific $A b s,{ }^{28,37}$ and the lack of Fas- $L$ detection using FACS or RT-PCR. ${ }^{21,35,38}$ We demonstrated Fas-L expression on escape tumor cell lines by FACS analysis, thus avoiding lymphocyte contamination that may contribute to Fas- $L$ detection in fresh tumor samples, by immunohistochemistry using a different antibody both on cytospins and on tumor samples after reinjection in vivo and by RT-PCR using specific primers and sequencing of the resulting fragment.

Further controversies arose when transplanted cells, engineered to express Fas- $L$ either by transgene expression, ${ }^{22,23}$ by transfection ${ }^{24}$ or by transduction with an adenoviral vector, ${ }^{21}$ were shown to exhibit a proinflammatory effect that resulted in a profound infiltration of neutrophils and in tumor rejection. This immunologic reaction was explained by the apoptotic death of $\mathrm{T}$ cells and other Fas-expressing cells and the production of IL-1 $\beta$ through their activated cascade of caspase proteases or of IL-8 by endothelial cells. ${ }^{39}$ These observations significantly differ from our results, as we failed to detect an inflammatory infiltration of neutrophils in growing escape tumors or after injection of escape tumor cells. This is consistent with our previous observation that tumor rejection was mediated by $T$ cells $^{26}$ and that apoptotic $T$ cells were specifically found in close proximity of Fas $-\mathrm{L}^{+}$ escape tumor cells. ${ }^{27}$ Moreover, we demonstrated here that Fas- $\mathrm{L}^{+}$escape tumor cell lines induced a Fas-dependent target cell apoptosis in vitro that was blocked with a soluble Fas-Fc fusion protein. These results indicate that Fas- $L$ on 
the tumor cell surface was functional and may have enabled tumor escape in vivo. The likely involvement of Fas- $L$ in the escape of tumor cells is further supported by our observation that as few as $0.5 \times 10^{6}$ injected escape tumor cells formed tumors in vaccinated mice (see Table 1 and Figure 3), indicating that these cells were not simply outnumbering the immune response, but displayed an active immunosuppressive mechanism in situ to escape the systemic rNeu-specific immune response. The significant delay of escape tumor progression in Balbc/neu vaccinated mice in comparison to Balbc/mock vaccinated mice may result from the different degree of Fas- $L$ expression on escape tumors. The faint Fas-L expression in $\mathrm{IHC}$ and FACS analysis of the spontaneous tumor cell line (NF9006) indicated that a Fas-L expression above a certain threshold was necessary for tumor cells to escape an $\mathrm{ASI}$

Tumor escape from immune response appears a complex mechanism that, besides Fas-L, likely involves other immunomodulatory factors, such as TGF- $\beta .{ }^{40}$ The availability of escape tumor cell lines constitutively expressing both a clinically relevant TAAg (rNeu) and Fas-L may help to clarify some of the controversies surrounding the involvement of Fas- $L$ in tumor escape mechanisms. ${ }^{20,36}$ In particular, protocols to prevent Fas-L expression or Fas-L-mediated escape in vivo could be investigated. This may be of relevance in future clinical trials of $\mathrm{ASI}$ in patients with breast cancer, as Fas-L expression was shown in various breast carcinomas and derived cell lines. ${ }^{16-18}$

\section{Materials and Methods}

\section{Mice}

MMTV/r-Neu FVB mice $\left(\mathrm{H}-2^{q}\right)$ transgenic for the rat neu protein (rNeuTG) were purchased from Charles River, Germany. Animal care was in accordance to institutional guidelines. Transgenic animals were identified by PCR for rNeu as described previously. ${ }^{26}$

\section{Cell lines}

Balb/c 3T3 $\left(\mathrm{H}-2^{\mathrm{d}}\right)$ fibroblasts transfected with a SV2-Neo-SP65 plasmid alone (Balbc/mock) or together with the pBR322-rNeu plasmid (Balbc/neu) have been described previously. ${ }^{26}$

\section{Establishment of spontaneous and escape tumor-derived cell lines}

Tumors were excised and minced into small pieces ( $<0.5 \mathrm{~mm}$ each) in PBS and incubated for $20 \mathrm{~min}$ at $37^{\circ} \mathrm{C}$ in the presence of Trypsin/ EDTA (Sigma, Buchs, Switzerland). The cell suspension was filtered through a $0.45 \mu \mathrm{m}$ cell strainer (Becton Dickinson, Heidelberg, Germany), washed in DMEM supplemented with $10 \%$ FCS, and grown in William's E medium supplemented with $10 \%$ FCS, antibiotics (penicillin/streptomycin), $2 \mathrm{mM}$ glutamin, and $10^{-5} \mathrm{M} \beta$-mercaptoethanol (all from Sigma). Established cell lines were then progressively adapted from William's E (Sigma) to DMEM medium. The $\mathrm{rNeu}^{+}$breast cancer cell line NF9006 was cloned from a spontaneous breast adenocarcinoma as described. ${ }^{41}$

\section{Vaccination protocols}

For vaccination/challenge experiments, mice were vaccinated and boosted (after 14 days) intraperitoneally (i.p.) with $5 \times 10^{6} \mathrm{Balbc} / \mathrm{neu}$ or Balbc/mock fibroblasts. Two weeks after the boost, mice were challenged by subcutaneous (s.c.) injection of $0.5-2 \times 10^{6} \mathrm{rNeu}^{+}$ spontaneous or escape tumor cells. Tumor formation and size was assessed every $3-4$ days using a calibrator. Tissues were collected for histological and immunohistochemical analysis at indicated time points.

\section{Immunohistochemistry}

Paraffin-embedded tissues were deparaffinized and pretreated by microwave heating in $10 \mathrm{mM}$ citrate buffer, $\mathrm{pH}$ 6.0. Slides were incubated with either a rabbit anti-HER-2/neu (Dako, Copenhagen, Denmark), a rabbit anti-Fas-L (N-20, Santa Cruz Biotechnology, Santa Cruz, CA, USA) or a rabbit anti-topoisomerase Il $\alpha$ (TOPOIIAp, Novocastra Laboratories, Newcastle, UK) polyclonal antibody (Ab) diluted in TCGA buffer (Tris $50 \mathrm{mM}, \mathrm{pH} 7.5$ supplemented with $1 \%$ casein, $0.02 \% \mathrm{NaN}_{3}$ and $5 \%$ normal goat serum). Alternatively, cytospins were incubated with a mouse anti-pan-cytokeratin (Sigma), a rabbit anti-Fas-L (N20) or rabbit anti-Fas (Oncogene Research, MA, USA) polyclonal Ab diluted in TCGA. After incubation with biotin-conjugated goat anti-mouse or goat anti-rabbit Ab (Dako), reactivities were detected using alkaline phosphatase-conjugated avidin-biotin complex (Dako) and Newfuchsin substrate (Sigma) according to manufacturer's instructions. Stainings were scored as described. ${ }^{27}$

\section{FACS analysis}

Cells were incubated at $4^{\circ} \mathrm{C}$ for $30 \mathrm{~min}$ with mouse anti-rNeu Ab-4 (Oncogene Science, Tarzana, CA, USA) or biotinilated anti-Fas-L ( $\mathrm{H} 11$, IgG2a from Alexis, Lausen, Switzerland), anti-H-2D ${ }^{q} / L^{q}$, anti-I- $A^{q}$, antiCD80 or anti-CD86 mAbs (IgG2a, all from Pharmingen, San Diego, CA, USA), washed with PBS, followed by incubation with FITC-conjugated Streptavidin or FITC-conjugated goat anti-mouse IgG (Southern Biotechnology, Birmingham, AL, USA) and analyzed for fluorescence on a FACScan (Becton Dickinson, Heidelberg, Germany).

\section{RT-PCR for Fas-L}

Total RNA was extracted from $5 \times 10^{6}$ tumor cells using the SVTotal RNA Isolation system (Promega, Madison, WI, USA) according to manufacturer's instructions. One $\mu \mathrm{g}$ RNA was subjected to reverse transcriptase reaction (RT) using $0.5 \mu \mathrm{g}$ oligo $\mathrm{p}(\mathrm{dT})_{15}$ primer, $0.4 \mathrm{mM}$ dNTP nucleotide mix (Roche Diagnostics, Rotkreuz, Switzerland) and the M-MLV reverse transcriptase (Promega) for $1 \mathrm{~h}$ at $42^{\circ} \mathrm{C}$. Total RNA extracts without reverse transcriptase was used as control ruling out DNA contamination. RT reaction was stopped by incubation at $95^{\circ} \mathrm{C}$ for $5 \mathrm{~min}$. CDNA was then subjected to $40 \mathrm{PCR}$ cycles $\left(1 \mathrm{~min}\right.$ at $95^{\circ} \mathrm{C}, 1 \mathrm{~min}$ at $62^{\circ} \mathrm{C}, 1.5 \mathrm{~min}$ at $72^{\circ} \mathrm{C}$ ) using intron-spanning primers: 5-CACTGACCCCTCTAAAGAAGAA$3^{\prime}$ (forward) and 5'-TTGAATACTGCCCCCAGGTA-3' (reverse). Presence of a 547-bp fragment was analyzed on an agarose gel. Fas-L fragment was further confirmed by sequencing (Microsynth $\mathrm{GmbH}$, Balgach, Switzerland).

\section{Co-culture DNA fragmentation assay (JAM test)}

Target Fas-transfected P815-4.2 (P815-Fas) and P815 wild type (P815-WT) cells were kindly provided by $\mathrm{K}$ Matsuura and $\mathrm{P}$ Vassalli, (Geneva, Switzerland). The cell death was quantitated by measuring 
target cell DNA fragmentation using the JAM test with some modifications. ${ }^{30,31}$ In brief, breast cancer cell lines (NF9006 and \#493) were seeded in flat bottom 96-well plates and incubated overnight. Thereafter, 5000 P815-WT or P815-Fas target cells were added that had been labeled by prior incubation with $2.5 \mu \mathrm{Ci} / \mathrm{ml}$ of $\left[{ }^{3} \mathrm{H}\right]$ thymidine at $37^{\circ} \mathrm{C}$ for $18-22 \mathrm{~h}$. After a coculture of $6-8 \mathrm{~h}$ cells were removed from the wells by adding $5 \mathrm{mM}$ EDTA and collected onto a glass fiber filter. The radioactivity of intact chromosomal DNA retained on filters was measured by liquid scintillation counting. Specific killing was calculated using the following equation:

$$
\% \text { Specific killing }=\left(\begin{array}{ll}
S & E / S
\end{array}\right) \times 100
$$

where $E$ (experimental) is c.p.m. of retained DNA in the presence of effector cells, and $S$ (spontaneous) is c.p.m. of retained DNA in target cells in the absence of effector cells. Soluble Fas- $L$ was obtained from supernatant of Neuro-2a cells stabily transfected with Fas- $L$ as described previously ${ }^{42}$ and kindly provided by A Fontana (Zurich, Switzerland). Where indicated Fas-Fc fusion protein was added to the cultures to inhibit Fas/Fas-L activity at a concentration of $20 \mu \mathrm{g} / \mathrm{ml}$ according to manufacturer's instructions (Alexis).

\section{Acknowledgements}

CD Gimmi is supported by the Swiss National Science Foundation 3244482.95. This work was supported by the Swiss National Science Foundation grant 3100-4038.96/1 and the Swiss Cancer League grant 268-1-1996. T Brunner is supported by the Swiss National Science Foundation grant 31-54079.98 and the Swiss Cancer League grant 6172-1998.

\section{References}

1. Fisher R (1998) Relapse after successful treatment with immunotherapy: lessons for the future. Cancer J. Sci. Am. 4: 78-79

2. Cromme F, Airey J, Heemels M, Ploegh H, Keating P, Walboomers JM, Meijer C and Stern PL (1994) Loss of transporter protein, encoded by the TAP-1 gene, is highly correlated with loss of HLA expression in cervical carcinomas. J. Exp. Med. 179: 335-340

3. Cohen E and Kim T (1994) Neoplastic cells that express low levels of MHC class I determinants escape host immunity. Sem. Cancer. Biol. 5: 419-428

4. Ye X, McCarrick J, Jewett $L$ and Knowles BB (1994) Timely immunization subverts the development of peripheral nonresponsiveness and suppresses tumor development in simian virus 40 tumor antigen-transgenic mice. Proc. Natl. Acad. Sci. USA 91: 3916-3920

5. Chen L, Linsley PS and Hellström KE (1993) Costimulation of T cells for tumor immunity. Immunol Today 14: 483-486

6. Huang AYC, Bruce AT and Pardoll DM (1996) Does B7-1 expression confer antigen-presenting cell capacity to tumors in vivo? J. Exp. Med. 183: 769-776

7. Townsend S and Allison J (1993) Tumor rejection after direct costimulation of CD8+ T cells by B7-transfected melanoma cells. Science 259: 368-370

8. Pawelec G, Zeuthen J and Kiessling R (1997) Escape from host-antitumor immunity. Crit. Rev. Oncol. 8: 111-114

9. Hahne M, Rimoldi D, Schroter M, Romero P, Schreier M, French L, Schneider P, Bornand T, Fontana A, Lienard D, Cerotini J and Tschopp J (1996) Melanoma cell expression of Fas (Apo-1/CD95) ligand: implications for tumor escape. Science 274: $1363-1366$

10. O'Connell J, O'Sullivan G, Collins J and Shanahan F (1996) The Fas counterattack: Fas-mediated T cell killing by colon cancer cells expressing Fas ligand. J. Exp. Med. 184: 1075-1082
11. Strand S, Hofmann WJ, Hug H, Mueller M, Otto G, Strand D, Nariani SM, Stremmel W, Krammer PH and Galk PR (1996) Lymphocyte apoptosis induced by CD95 (Apo-1/Fas) ligand-expressing tumor cells - A mechanism of immune evasion? Nature Med. 2: 1361-1366

12. Bennett M, O'Connell J, O'Sullivan G, Brady C, Roche D, Collins J et al (1998) The Fas counterattack in vivo: apoptotic depletion of tumor-infiltrating lymphocytes associated with Fas ligand expression by human esophageal carcinoma. J. Immunol. 160: 5669-5675

13. ShirakiK, Tsuji N, Shioda T, IsselbacherKand TakahashiH (1997) Expression of Fas ligand in liver metastases of human colonic adenocarcinoma. Proc. Natl. Acad. Sci. USA 94: 6420-6425

14. Niehans G, Brunner T, Frizelle S, Liston J, Salerno C, Knapp D, Green DR and Kratzke RA (1997) Human lung carcinomas express Fas ligand. Cancer Res. 57: $1007-1011$

15. O'Connell J, Bennett M, O'Sullivan G, O'Callaghan J, Collins J, Shanahan F (1999) Expression of Fas ligand (CD95/APO-1) by human breast cancers: significance for tumor privilege. Clin. Diagn. Lab. Immunol. 6: 457-463

16. Münschen M, Moers $C$, Warskulat U, Even J, Niederacher D and Beckmann M (2000) CD95 ligand expression as a mechanism of immune escape in breast cancer. Immunology 99: 69-77

17. Mullauer L, Mosberger I, Grusch M, Rudas M and Chott A (2000) Fas-ligand is expressed in normal breast epithelial cells and is frequently upregulated in breast cancer. J. Pathol. 190: 20-30

18. Herrnring C, Reimer T, Jeschke U, Makovitzky J, Kruger K, Gerber B, Kabelitz D and Friese K (2000) Expression of the apoptosis-inducing ligands FasL and TRIAL in malignant and benign human breast cancer. Histochem. Cell. Biol. 113: $189-194$

19. O'Connell J, Bennett M, O'Sullivan G, Collins J and Shanahan F (1999) Fas counter-attack - the best form of tumor defense. Nature Med. 5: 267-268

20. Igney $F$, Behrens $C$ and Krammer $P(2000)$ Tumor counterattack-concept and reality. Eur. J. Immunol. 30: 725-731

21. Arai H, Gordon D, Nabel E and Nabel G (1997) Gene transfer of Fas ligand induces tumor regression in vitro. Proc. Natl. Acad. Sci. USA 94: 13862 - 13867

22. Allison J, Georgiou H, Strasser A and Vaux D (1997) Transgenic expression of CD95 ligand on islet beta cells induces a granulocytic infiltration but does not confer immune privilege upon islet allografts. Proc. Natl. Acad. Sci. USA 94: 3943-3947

23. Kang S, Schneider D, Lin Z, Hanahan D, Dicker D, Stock P and Baekkeskov S (1997) Fas ligand expression in islets Langerhans does not confer immune privilege and instead targets them for rapid destruction. Nature Med. 3: 738-743

24. Seino K, Kayagaki N, Okumura K, Yagita H (1997) Antitumor effect of locally produced CD95 ligand. Nature Med. 3: 165-170

25. Muller WJ, Sinn E, Pattengale PK, Wallace R and Leder P (1988) Single-step induction of mammary adenocarcinoma in transgenic mice bearing the activated c-neu oncogene. Cell 54: 105-115

26. Céfai D, Morrison B, Skell A, Favre L, Balli M, Leunig M and Gimmi CD (1999) Targeting HER-2/neu for active-specific immunotherapy in a mouse model of spontaneous breast cancer. Int. J. Cancer 83: 393-400

27. Céfai D, Favre L, Buri C, WattendorfE, Marti A, Jaggi Rand Gimmi CD (2001) The role of Fas ligand expression in promoting escape from immune rejection in an in vivo spontaneous tumor model. Int. J. Cancer. 91: 529-537

28. Smith D, Sieg S and Kaplan D (1998) Technical Note: Aberrant detection of cell surface Fas ligand with anti-peptide antibodies. J. Immunol. 160: 4159-4160

29. Suda T, Takahashi T, Golstein P and Nagata S (1993) Molecular cloning and expression of the Fas ligand, a novel member of the tumor necrosis factor family. Cell $75: 1169-1178$

30. Matzinger $P(1991)$ The Jam test: a simple assay for DNA fragmentation and cell death. J. Immunol. Methods 145: 185-192

31. Böhm C, Hanski M, Gratchev A, Mann B, Moyer M, Riecken E and Hanski C (1998) A modification of JAM test is necessary for correct determination of apoptosis induced FasL+ adherent tumor cells. J. Immunol. Methods 217: 71 78

32. Van Parijs V and Abbas A (1998) Homeostasis and self-tolerance in the immune system: turning lymphocytes off. Science 280: 243-248

33. Bellgrau D, Gold D, Selawry H, Moore J, Franzusoff A and Duke R (1995) A role for CD95 ligand in preventing graft rejection. Nature 377: 630-632 
34. Griffith T, Brunner T, Fletcher S, Green D and Ferguson T (1995) Fas ligandinduced apoptosis as a mechanism of immune privilege. Science 270: 1189 1192

35. Chappell D, Zaks T, Rosenberg S and Restifo N (1999) Human melanoma cells do not express Fas (Apo-1/CD95) ligand. Cancer Res. 59: 59-62

36. Restifo N (2000) Not so Fas: Re-evaluating the mechanisms of immune privilege and tumor escape. Nature Med. 6: 493-495

37. Gastman B, Atarshi Y, Reichert T, Saito T, Balkir L, Rabinowitch Hand Whiteside TL (1999) Fas ligand is expressed on human squamous cell carcinomas of the head and neck, and it promotes apoptosis of T lymphocytes. Cancer Res 59: 5356-5364

38. Favre-Felix N, Fromentin A, Hammann A, Solary E, Martin F and Bonnotte B (2000) The tumor counterattack hypothesis revisited: Colon cancer cells do not induce T cell apoptosis via fas (CD95, APO-1) pathway. J. Immunol. 164: 50235027
39. Miller D (1997) The role of the caspase family of cystein proteases in apoptosis. Semin. Immunol. 9: 35-49

40. Chen J, Sun Y and Nabel G (1998) Regulation of proinflammatory effects of Fas ligand (CD95L). Science 282: 1714-1717

41. Morrison BW and Leder $P$ (1994) neu and ras initiate murine mammary tumors that share genetic markers generally absent in c-myc and int-2 initiated tumors. Oncogene 9: 3417-3426

42. Schneider P, Holler N, Bodmer J, Hahne M, Frei K, Fontana A and Tschapp J (1998) Conversion of membrane-bound Fas (CD95) ligand to its soluble form is associated with downregulation of its proapoptotic activity and loss of liver toxicity. J. Exp. Med. 187: 1205-1213 